\title{
PLANETARY SEARCHES USING OPTICAL ASTROMETRIC INTERFEROMETERS
}

D. H. Staelin and M. M. Colavita

Massachusetts Institute of Technology

Cambridge, Massachusetts 02139

\section{Shao}

Smithsonian Astrophysical Observatory

Cambridge, Massachusetts 02138

ABSTRACT. Detection of planets by virtue of their gravitational perturbations of nearby visible stars generally requires relative astrometric accuracies of $\sim 3 \times 10^{-4}$ arc sec for Jovian planets and $\sim 3 \times 10^{-7}$ arc sec for terrestrial planets. Two-color optical astrometric interferometers on earth should be capable of $\sim 10^{-4}$ arc sec rms accuracy for stars brighter than magnitude 7-10, while small one-color space systems should achieve at least $\sim 10^{-5}$ arc sec. Two-color systems permit correction every few milliseconds for the random wavefront tilt due to atmospheric turbulence; the correction is based on the observed offset between the red and blue fringes, and the known atmospheric dispersion. A 3.4-meter baseline interferometer on Mount Wilson has demonstrated $\sim 0.02$ arc sec rms fluctuations for 1-second samples.

\section{DETECTABILITY OF PLANETS}

Planets are detectable by virtue of their periodic perturbation of the position of a central observable star. The angular displacement of the star depends on the relative masses of the planet and the star, and on their distances from each other and from the earth. This simple relationship is expressed in Figure 1 in terms of the rms astrometric accuracy $(1 \sigma)$ required to detect the $4 \sigma$ peak-to-peak displacement of a star at period T; this sensitivity is displayed as a function of the mass $M$ of the planet relative to the mass $\mathrm{M}_{\mathrm{J}}$ of Jupiter. Since this relationship also depends on the stellar mass and distance, the masses and distances for the thirty nearest stars brighter than 9 th magnitude were used to deduce the average sensitivity requirements illustrated in the figure; the one-sigma variation in these sensitivities for these stars is also indicated in the figure together with the corresponding sensitivity requirements for Doppler-shift measurements of periodic stellar motion.

The figure suggests that Jupiter and Saturn could be detected around typical nearby stars with astrometric accuracies between $10^{-3}$ and $10^{-4}$ arc sec, or with Doppler accuracies between 1 and $10 \mathrm{~m} \mathrm{~s}^{-1}$. Terrestrial planets would require accuracies between $10^{-6}$ and $10^{-7}$ arc $\mathrm{sec}$, which is extreme, even for interferometers in space. 


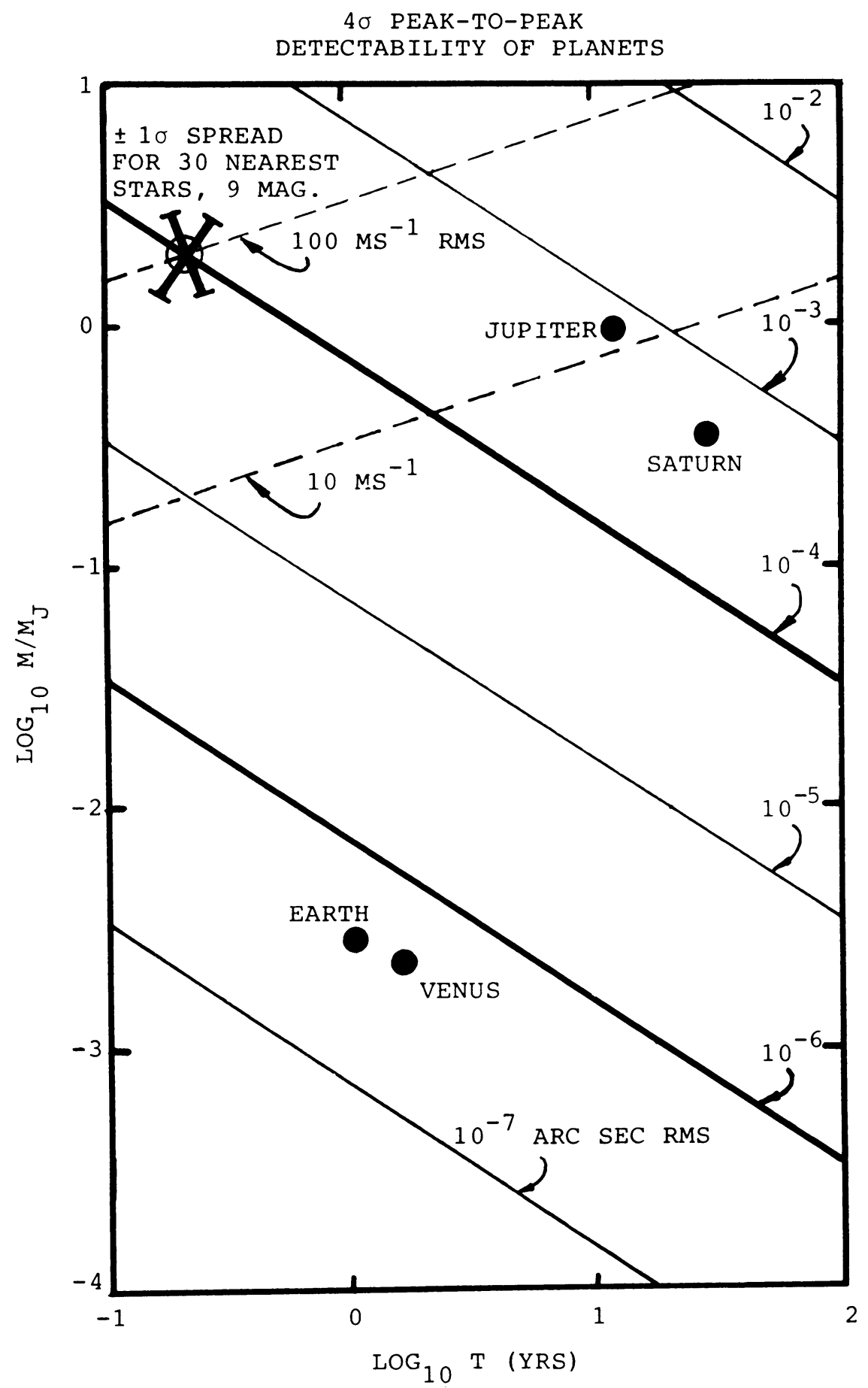

Figure 1. Detectability of nearby planets as a function of planetary period $\mathrm{T}$ and mass $\mathrm{M}$ relative to the mass of Jupiter $\mathrm{M}_{\mathrm{J}}$. 


\section{PRINCIPLES OF ASTROMETRIC INTERFEROMETRY}

The basic principles of two-color astrometric interferometry were first described by Shao and Staelin (1977) and are summarized here.

The basic configuration is that of a conventional Michelson stellar interferometer with two co-planar interfering beams focused on a photodetector. By using half-octave bandwidths a white fringe plus several smaller fringes are obtained. When the length of one of the two interferometer arms is varied, the detected photon count varies as the central fringe is thus scanned. By systematically scanning the white fringe at $\sim 500 \mathrm{~Hz}$ it is possible to track its random motion due to the atmosphere or other causes. These changes in arm length can be monitored with rms errors of several angstroms, and thus the instantaneous effective angle of arrival of the starlight can be monitored with great accuracy (100 angstroms for a 2-meter baseline corresponds to $10^{-3}$ arc $\mathrm{sec})$. The first successful use of a one-color interferometer to track broadband stellar fringes was reported by Shao and Staelin (1980).

The atmosphere introduces several problems, however. First, the absolute atmospheric refraction is not known sufficiently accurately. This problem is solved by effectively placing the interferometer in a vacuum so the systematic refractive effect of the planar atmosphere overhead is negligible. This is accomplished in the Mount Wilson interferometer by placing the moveable delay line in vacuum such that the two air paths from star to photodetector are always equal.

The random tilt of the arriving wavefront due to atmospheric turbulence is more troublesome and normally limits all ground-based optical astrometric systems to accuracies no better than $10^{-1}-10^{-2}$ arc sec per night unless the stellar separations being measured are very sma11. A two-color astrometric interferometer measures the positions of the red and blue fringes separately every $1-4 \mathrm{msec}$, and from the offset between them the tilt contributed by the atmosphere can be estimated by virtue of the known dispersion of the atmosphere. In general the instantaneous atmospheric offsets of the white fringe are approximately 60-120 times the offset between the blue and the red fringes, the blue being displaced more. This ratio depends on the blue and red spectra, which vary with stellar type, weather, and detector characteristics, and can be determined a priori or by subsequent analysis of the red and blue fringe data.

Although random fringe position errors are multiplied by a factor of $\sim 100$, averaging such independent measurements over even one second generally permits accuracies superior to one-color astrometry.

The largest single source of error in two-color astrometry is due to water vapor, because its contributions to dispersion are quite different from those of temperature and density. High dry sites therefore have significant advantages, and improvements of a factor of ح 5-10 relative to one-color measurements are anticipated. Observed improvements of a factor of at least $\sim 3$ are illustrated in Figure 2, as discussed in the next section.

Stars $5-10^{\circ}$ apart might be measured with accuracies of $10^{-3}$ arc second after $\sim 1$ hour for a 20 -meter baseline. The $10^{-4}$ arc second accuracy needed for $4 \sigma$ detection of Jovian planets might require 

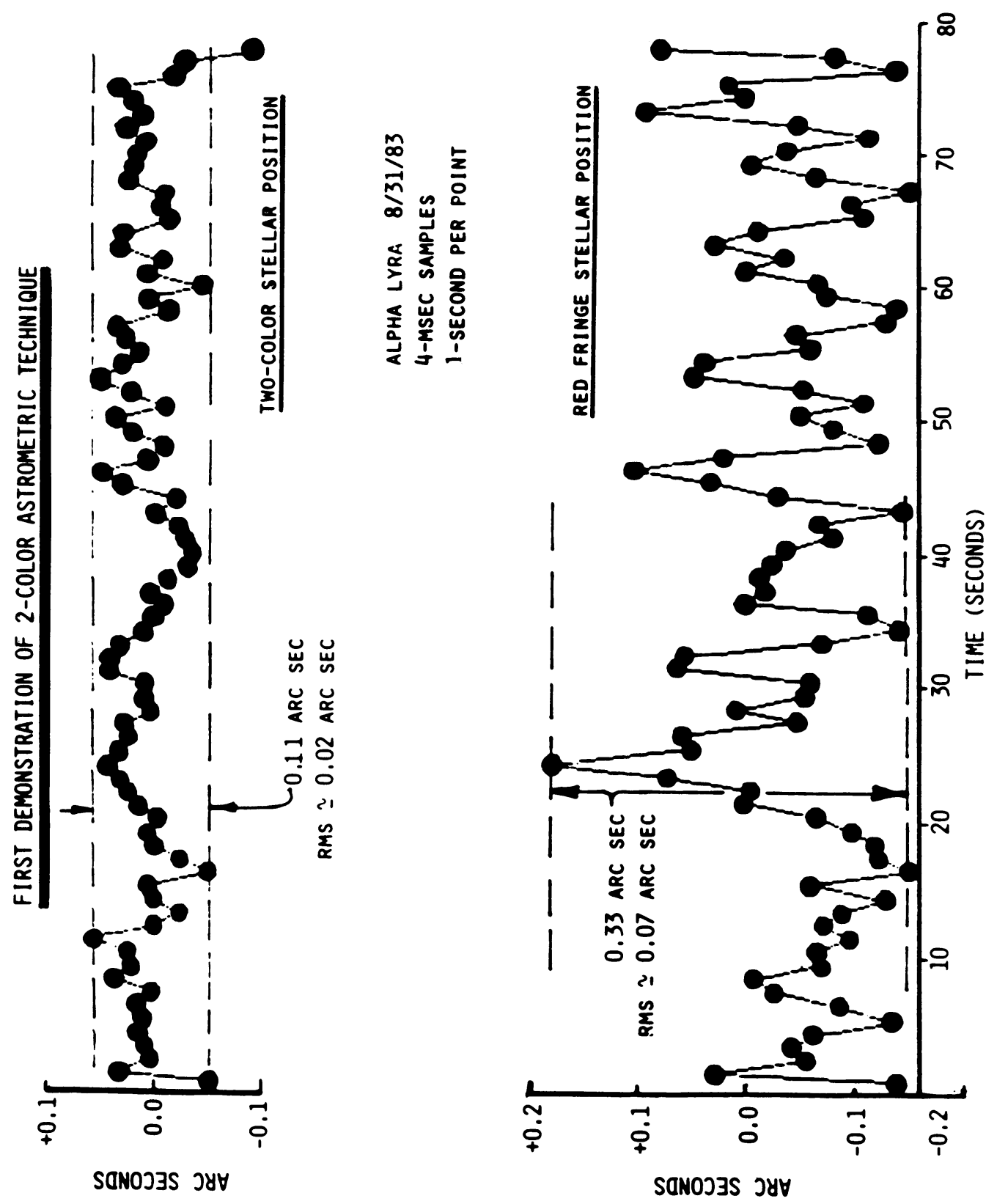

Figure 2. Stellar positions observed with an astrometric interferometer; effects of terrestrial rotation have been empirically removed. 
several months of averaging. A dry site like Mauna Kea might reduce this requirement. More observations and analysis are required to refine these estimated limits to performance.

The other requirement for astrometric accuracy is known baseline coordinates. By relating via lasers the mechanical axes to bedrock, and by observing many stars in sequence, both north-south and eastwest, the impact of the baseline coordinates can be reduced to an anticipated accuracy per night of $\sim 10^{-3}$ arc sec.

\section{OBSERVATIONS WITH THE ASTROMETRIC INTERFEROMETER AT MOUNT WILSON}

The Mark-II interferometer has a 3.4-meter north-south baseline and operates in two colors with $\sim 3-\mathrm{cm}$ apertures. It can presently track stars brighter than magnitude $\sim 3$. In Figure 2 are shown data for Alpha Lyra obtained August 31, 1983 using 4-msec integration times. The star was observed for 80 seconds and the strong influence of terrestrial rotation was removed empirically. The residuals are presented as a sequence of one-second averages showing the stabilities of the one-color and two-color interferometric techniques.

The rms fluctuations of $\sim 0.07$ arc sec for these one-second onecolor measurements are testimony to the extremely good seeing of the Mount Wilson site. The two-color technique has reduced these fluctuations by a factor of $\sim 3$ here. The two-color technique leaves residual errors with a non-white spectrum. Because the baseline stability of the Mark-II was quite limited, it is not yet known what fraction of the $\sim 0.02$ arc sec rms errors for these 1-second two-color measurements is due to water vapor, and what fraction is due to mechanical instabilities or other causes.

\section{SPACE-BASED OPTICAL ASTROMETRIC INTERFEROMETRY}

In space larger mirrors and longer integration times can be used; 30-cm mirrors should permit searches for stars brighter than $\sim 16-20$ magnitude. At least three stars should be observed simultaneously, two of which merely establish the haseline with respect to which the third target star is measured.

One conceptual design would be a free-flyer $\sim 3.5 \times 3.5 \times 1$ meter slowly rotating about an axis oriented toward the sky sector currently being observed. This should result in stellar separation accuracies of $\sim 10^{-5}$ arc sec, and possibly even $\sim 10^{-6}$ arc sec in special cases.

This work was supported in part by NSF Grant AST-7919553, NASA Grant NAG 2-50, ONR Contract N00014-80-C-0348, NRL Contract N00014-84$\mathrm{C}-2082$, and by the MIT Sloan Fund. We also appreciate the assistance and collaboration of C. Papa, P. Garnavich, S. Knowles, K. Johnston, and the staff of the Mount Wilson Observatory.

\section{REFERENCES}

M. Shao and D. Stae1in, J. Opt. Soc. Am. 67, 81 (1977). , Applied Optics, 19, 1519 (1980). 